\title{
FOXN3 Inhibits Cell Proliferation though Regulating E2F1 in Prostate Cancer
}

\author{
NING. ZHANG, HAIJUN. YANG* AND QINGQI. ZENG \\ Department of Traditional Chinese Medicine, Jiangsu Health Vocational College, No. 69 Huangshanling Road, Nanjing, Jiangsu \\ 211800, China
}

\section{Zhang et al.: FOXN3 Inhibits Cell Proliferation in Prostate Cancer}

\begin{abstract}
In this study, it was demonstrated that forkhead box N3 was downregulated in prostate cancer tissues compared to the matched normal adjacent tissues. Prostate cancer patients with high forkhead box N3 expression more frequently had longer survival $(\mathrm{P}<0.05)$ than those with low forkhead box $\mathrm{N} 3$ expression. Forkhead box N3 inhibited prostate cancer cell proliferation in vitro and in vivo. In addition, forkhead box $\mathrm{N} 3$ repressed expression of $\mathrm{E} 2 \mathrm{~F} 1$, a reported potential oncogene at the messenger ribonucleic acid and protein levels. Taken together, these data delineate an unrecognized function of forkhead box N3 as a tumor suppressor in prostate cancer via repressing the expression of E2F1.
\end{abstract}

Key words: Prostate cancer, FOXN3, E2F1, cell proliferation

As a common urogenital system malignant tumor in men, prostate cancer (PC) threatens men's health and becomes an important cause of death in the elderly ${ }^{[1]}$. The incidence of PC has increased in most countries and $\mathrm{PC}$ represents a major disease burden worldwide in the past decade ${ }^{[2,3]}$. Treatment options for PC depended on clinicopathological features, such as Gleason score, TNM stage, serum prostate-specific antigen (PSA) and surgical margin status. However, these factors explain only a moderate proportion of the observed heterogeneity in treatment outcome ${ }^{[4]}$. Therefore, the urgent need is to better elucidate pathogenesis underlying the progressive PC and to identify more accurate predictors for patients, so that optimal therapeutic strategies can be decided. Forkhead box N3 (FOXN3), also named checkpoint suppressor 1 (CHES1), was first identified in the yeast as a check point defects suppressor ${ }^{[5]}$. FOXN3 is a member of the forkhead box transcription factors family ${ }^{[6]}$. More than 100 members of the forkhead box family are classified into 15 subclasses (FOXA through FOXS $)^{[6]}$. The forkhead domain, also known as winged helix is the common DNA-binding domain of these proteins. The forkhead box family has been implicated in a broad spectrum of cellular processes, metabolism, DNA repair, differentiation, including cell proliferation, and aging ${ }^{[7]}$. As a FOXN subfamily member, FOXN3 is required for Xenopus laevis craniofacial and eye development ${ }^{[8]}$. A previous study showed that FOXN3 could down-regulate E2F5 in human cells to control cell cycle or inhibit protein biosynthesis ${ }^{[6]}$. In addition, studies have shown that FOXN3 is significantly downregulated in several cancer tissues including oral laryngeal carcinoma hepatocellular carcinoma, squamous cell carcinoma and colon cancer ${ }^{[6,9,10]}$. Based on these reports, it was surmised that as a transcription factor FOXN3 played a role in progression of human cancer by regulating other genes. In our research, cell cycle-specific transcription factor, E2F1 was identified as a downstream gene of FOXN3. E2F1 was originally identified as a member of the E2F transcription factors family ${ }^{[11]}$. E2F family, which was named for its function, regulates transcription by binding to the $\mathrm{E} 2 \mathrm{~F}$ site (TTTSSCGC: $\mathrm{S}=\mathrm{C}$ or $\mathrm{G}$ ). E2F family was originally regarded as an activator to the promoter of adenovirus E2 in the 1980s. With the in-depth study, it was found that the E2F family played an key role in the process of cell growth, differentiation and apoptosis ${ }^{[12]}$. Exogenous E2F expression is growth inhibitory before, during, and after cellular transformation ${ }^{[13]}$. Additionally, E2F family can regulate the cell cycle $\mathrm{G} 1$ to $\mathrm{S}$ phase transition $^{[14]}$. E2F1, a member of the E2F family, accumulates the integrated signal of the $\mathrm{G} 1 / \mathrm{S}$ transition regulators, and is required for cell proliferation ${ }^{[15]}$. By contrast, studies have showed that E2F1 inducing apoptosis is a kind of protective mechanism against tumor cell proliferation ${ }^{[16]}$. Therefore, E2F1 may have 
opposite activities in different cancer. Another recent study found that E2F1 knockdown inhibited prostate tumor growth through sensitizing tumor cells to ICAM1 mediated antiimmunity by NF- $\mathrm{KB}$ modulation in vitro and in vivo ${ }^{[17]}$. In the present work, FOXN3 expression was shown to significantly downregulate E2F1 in PC tissues compared with their matched normal adjacent tissues. By functional tests, it was demonstrated that FOXN3 could inhibit the proliferation of PC cells in vitro and in vivo by the possible underlying mechanism. Therefore, this study improved the understanding of the role for FOXN3 in PC, which could represent a new approach for the prevention and therapy of this cancer. The tissue samples prepared by pathologists are snap frozen and stored at $-80^{\circ}$ subsequently. The present study was approved by the Research of the Jiangsu Health Vocational College. Informed consent was obtained from all the patients. The collection of fresh tumor tissue samples for clinical analysis was approved by the Universal Ethics Committee of the Jiangsu Health Vocational College. Total RNA was isolated from PC cell lines or PC tissues using Trizol reagent (Invitrogen). Subsequently, cDNAs were reverse-transcribed from total RNAs with the RT reagent kit (TaKaRa, Shiga, Japan). Quantitative RTPCR (Q-PCR) was used for gene expression by using SYBR PCR master mix in the ABI Step One-Plus Detection system (Applied Biosystems, USA) according to the manufacturer's introductions. The PCR primers used were as follows, FOXN3-F: 5'-ACTCTGACATGCCCTACGATG-3', FOXN3-R: 5'-TCTGACTCCTCTCTTTGTCCAC-3'; Ki67-F: 5'-GGGCCAATCCTGTCGCTTAAT-3', Ki67-R: 5'-GTTATGCGCTTGCGAACCT-3'; PCNA-F: 5'-CCTGCTGGGATATTAGCTCCA-3', PCNA-R: 5'-CAGCGGTAGGTGTCGAAGC-3'; E2F1-F: 5'-ACGCTATGAGACCTCACTGAA-3', E2F1-R: 5'-TCCTGGGTCAACCCCTCAAG-3'; $\beta$-actin-F:5'GTAAAGACCTCTATGCCAACA-3', $\beta$-actin-R: 5'-GGACTCATCCTACTCCTGCT-3'. The gene level was normalized to $\beta$-actin. Relative gene expression quantifications were calculated according to the comparative CT method. Each assay was performed in triplicate. PC-3, Du145 and LNCap were obtained from the American Type Culture Collection (ATCC, Manassas, VA, USA) and were cultured at $37^{\circ}$ in a humidified atmosphere with 5\% CO2. All cell lines were incubated in RPMI-1640 medium (Cellgro, Manassas, VA, USA) supplemented with $10 \%$ fetal bovine serum (FBS, Cellgro) and $1 \%$ penicillin/ streptomycin (Sigma, USA). The full-length human
FOXN3 gene sequences were cloned into pWPXL at the MluI and EcoRI sites. The reconstructed plasmid was verified by Sanger sequencing. To get lentivirus, lentiviral plasmid carrying FOXN3, along with lentiviral packaging mix in DMEM medium (Gibco, USA) were cotransfected into HEK 293NT cells. The culture medium were collected at $48 \mathrm{~h}$ after transfection, and then filtered by a $0.45 \mu \mathrm{m}$ filter, incubated with polyethylene glycol 8000 (PEG 8000) for $12 \mathrm{~h}$ before centrifugation $\left(4000 \times \mathrm{g}\right.$ for $20 \mathrm{~min}$ at $4^{\mathrm{o}}$; Thermo $)$. Lentivirus particles were stored at $-80^{\circ}$ until use. An empty backbone vector was used as a control. They were individually added to PC cells in 6-well plates at a MOI of 20. The full-length human E2F1 gene sequences were purchased from Addgene (pMax-E2F1, Plasmid \#16007). Plasmids were transfected into cells using Lipofectamine 2000 (Invitrogen) according to the manufacturer's instructions. Proteins were extracted from tissue or cell lines lysates, fractioned by $10 \%$ SDS-PAGE, and transferred to nitrocellulose membranes (Millipore, Massachusetts, USA). The antihuman FOXN3 polyclonal antibody (1:100, Ab50756) and the antihuman E2F1 polyclonal antibody (1:200, Ab64161) were purchased from Abcam (Cambridge, UK). The antihuman $\beta$-actin antibody (A3854) was purchased from Sigma-Aldrich (Missouri). Suspension of 2,000 cells were plated on a 96-well plate and placed at $37^{\circ}$ with $5 \% \mathrm{CO} 2$. Cells in each well were added with $20 \mu \mathrm{l}$ of MTT reagent $(5 \mathrm{mg} / \mathrm{ml}$, Sigma-Aldrich, USA) and incubated for $4 \mathrm{~h}$ at $37^{\circ}$ at different times. Then, optical density (OD) values at $570 \mathrm{~nm}$ were measured on a microplate reader for the cell proliferation assay. Each group was plated in 3 wells and was repeated 3 times. PC cells at a density of 1000 were plated in 6-well plates (Thermo, Massachusetts, USA) and cultured for $2 \mathrm{w}$. The colonies were fixed with $4 \%$ paraformaldehyde and then stained with $1 \%$ crystal violet. The colonies containing over 50 cells were counted and photographed. In the present study, animal experiments were performed in compliance with the guidelines of the Institute for the Jiangsu Health Vocational College. Six to 8-w old $\mathrm{BALB} / \mathrm{c}(\mathrm{nu} / \mathrm{nu})$ were obtained from the Shanghai Laboratory Animal Center (Shanghai, China) and group-housed at SPF facility under 12-h light-dark cycle at an environmental temperature of $20-25^{\circ}$. Mice were injected with suspended PC cells $\left(5 \times 10^{6} \mathrm{PC} 3 /\right.$ LNCap cells). Tumor sizes were measured every $3 \mathrm{~d}$. The mice were sacrificed after $2 \mathrm{w}$. Statistical analysis used the SPSS 19.0 statistical software. The product limit (Kaplan-Meier) method was used for the $7-y$ 
overall survival rate analysis after surgery as the observation indicator. The log rank test was used for a comparison of survival rates. Spearman's correlation analysis was used to analyze the correlation between FOXN3 and E2F1. All values are presented as the mean \pm SEM of three independent experiments. Statistical analysis was performed using an unpaired student's $t$ test. $P$ value of $<0.05$ was determined to be statistically significant. To investigate the general function of FOXN3 in PC, the mRNA level of FOXN3 in 50 pairs of $\mathrm{PC}$ tissues and matched non-tumorous prostatic tissues was determined by quantitative RTPCR (qRT-PCR). The result showed that FOXN3 was down-regulated in PC tissues compared with the adjacent non-cancerous tissues $(P<0.05)$ (fig. 1A). Survival analysis of the 253 PC patients, who lived longer 6 mo and less than $7 \mathrm{y}$, showed that high FOXN3 expression in PC patients was closely associated with good prognosis ( $\mathrm{n}=253$, log-rank test $P=0.050$, (fig. $1 \mathrm{~B}$ and 1C). Consistent with studies of FOXN3 in other cancers, these results implied that FOXN3 works as a tumor suppressor to inhibit in the PC development. To elucidate the role of FOXN3 in PC, the effects of
FOXN3 over-expression on PC cell proliferation was evaluated in vitro. First, we constructed a lentivirus vector containing the complete ORF of FOXN3 and established PC3-FOXN3, Du145-FOXN3 and LNCapFOXN3 cell lines. Both mRNA and protein levels of FOXN3 in PC3-FOXN3, Du145-FOXN3 and LNCapFOXN3 cell lines were significantly upregulated (fig. 2A and 2B). The effect of FOXN3 on cell proliferation were performed to assess by the MTT and colony formation assays. The results indicated that FOXN3 overexpression inhibited PC cell growth in vitro (fig. $2 \mathrm{C}$ and $2 \mathrm{D}$ ). Then the nude mouse model was constructed to further evaluate the role of FOXN3 in PC tumorigenesis by subcutaneous injection of PC3FOXN3 and LNCap-FOXN3 cells in vivo. Compared to the control group, the volume of tumors was significantly smaller in the PC3-FOXN3 and LNCapFOXN3 group (fig. 3A and 3B). The mass of tumors in PC3-FOXN3 and LNCap- FOXN3 group mice was much lighter than that of control group mice (fig. 3C). The expression of FOXN3 in xenografs was much higher in the PC3-FOXN3 and LNCap- FOXN3 group than the control group at the protein level (fig. 3D).
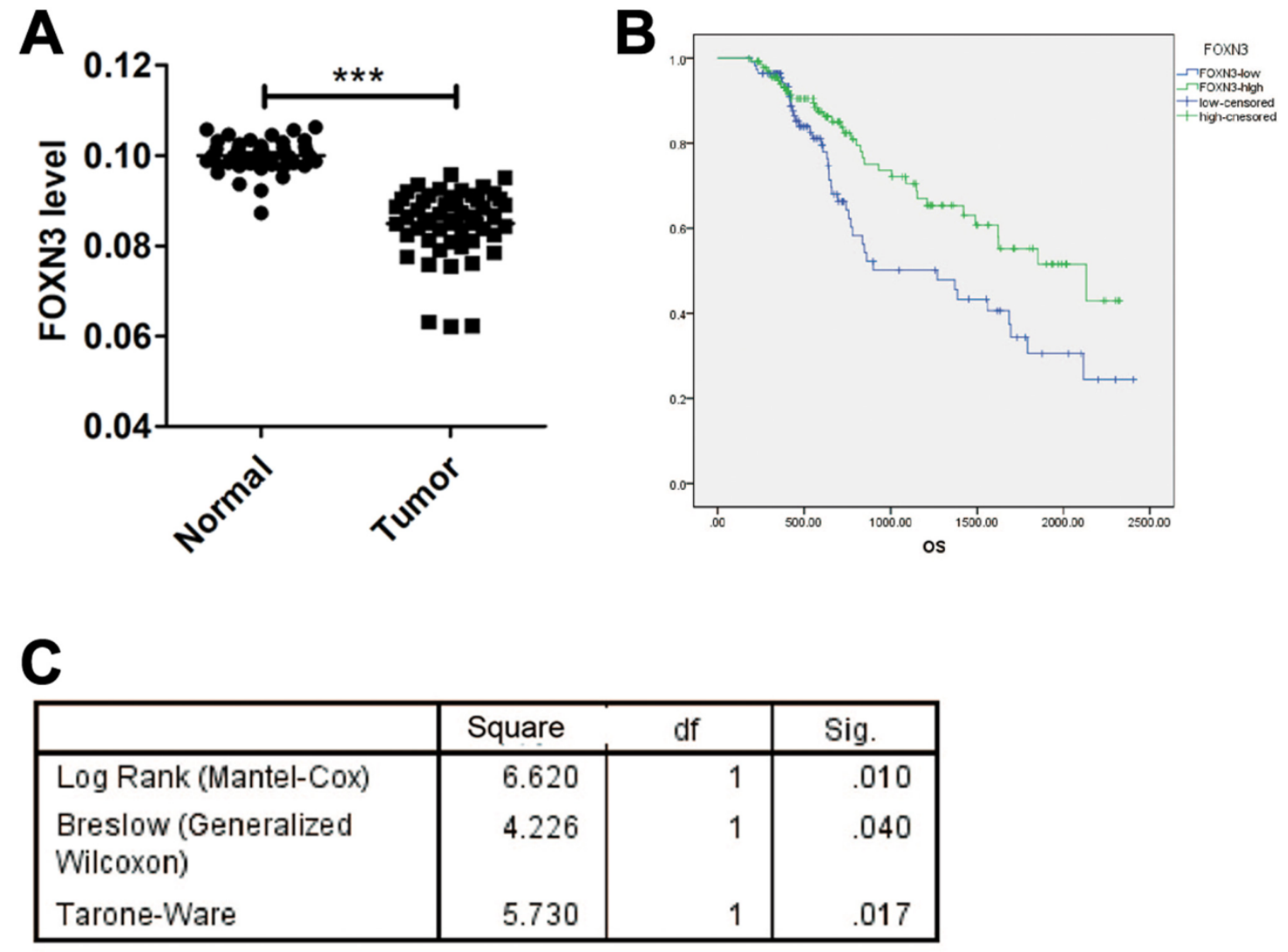

Fig. 1: FOXN3 is downregulated in $\mathrm{PC}$ tissues and associated with overall survival

(A) The mRNA level of FOXN3 in human PC tissues compared to non-cancer normal. $N=50$. (B, C) Kaplan-Meier analysis of the correlation between FOXN3 expression and overall survival in PC patients. $N=253$. Log-rank tests were used to determine statistical significance. 
www.ijpsonline.com
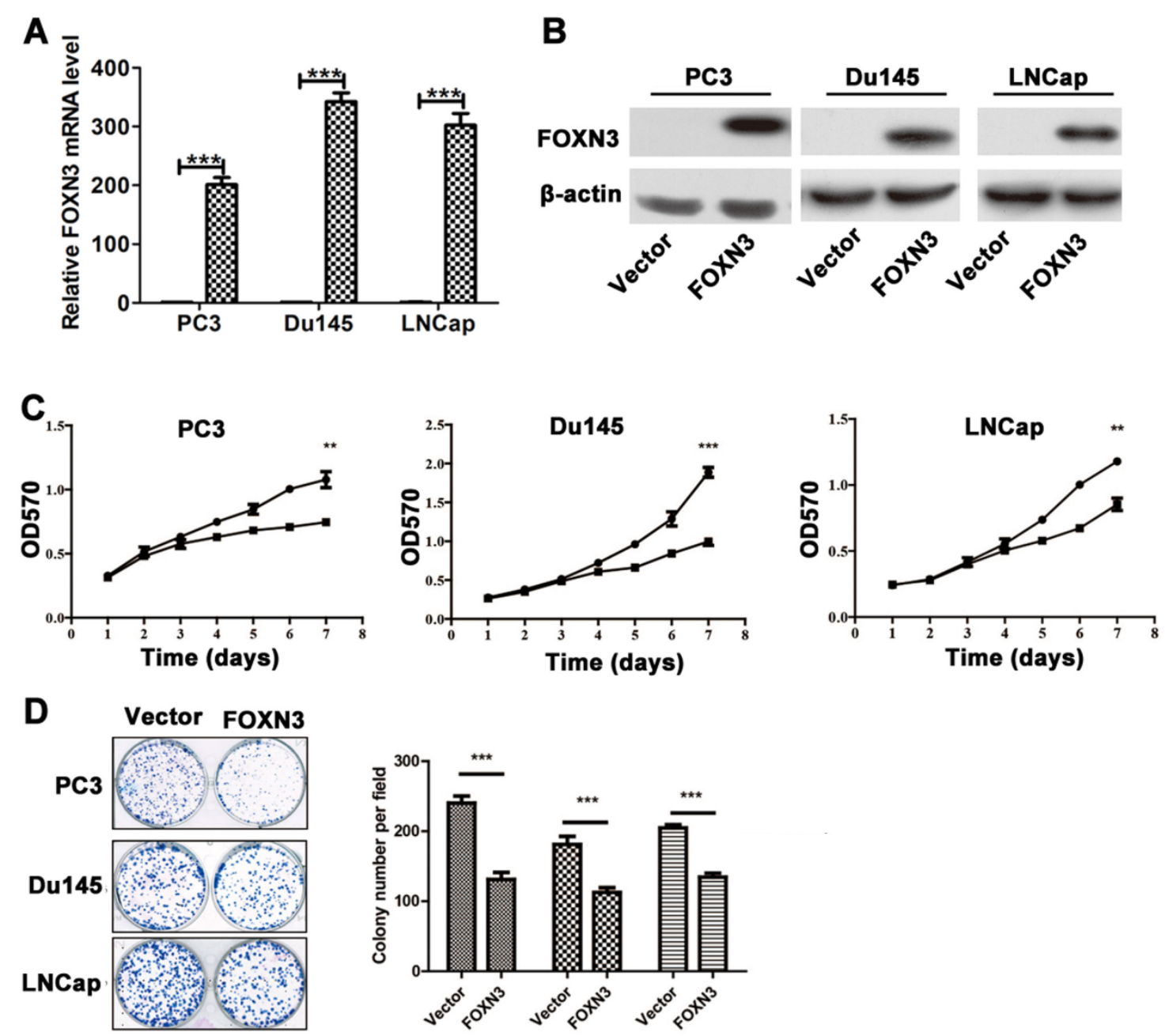

Fig. 2: FOXN3 overexpression inhibited $P C$ cell proliferation in vitro

(A) The relative mRNA level of FOXN3 in PC3, Du145 and LNCap cells after infected with lentivirus containing FOXN3 ORF. (B) The protein level of FOXN3 in PC3, Du145 and LNCap cells after infected with lentivirus containing FOXN3 ORF. (C) The cell proliferation was investigated when PC3, Du145 and LNCap cells infected with lentivirus containing FOXN3 ORF. (D) Colony formation assay was performed on in PC3, Du145 and LNCap cells infected with lentivirus containing FOXN3 ORF. $* * P<0.05, * * * P<0.01$.

Importantly, the proliferation markers (Ki67 and PCNA) were significantly downregulated in in the PC3-FOXN3 and LNCap- FOXN3 group (fig. 3E). $\mathrm{E} 2 \mathrm{~F} 5$, a member in E2F family, was reported as a downstream of FOXN3 ${ }^{[6]}$. With that in mind, the E2F family members were chosen to investigate the molecular mechanisms underlying the inhibitory effect of FOXN3 on PC cell proliferation. E2F1 was reported as a oncogene in $\mathrm{PC}^{[17,18]}$. Then, it was confirmed that the E2F1 mRNA and protein expression in PC3FOXN3, Du145-FOXN3 and LNCap-FOXN3 cell lines were significantly downregulated (fig. 4A and 4B). The expression of E2F1 mRNA in 50 pairs of PC tissues by qRT-PCR was measured to find that compared to nontumor prostatic tissues, E2F1 was upregulated in PC tissues (fig. 4C). Additionally, it was also detected correlation between E2F1 and FOXN3 in 373 PC tissues and found that E2F1 was significantly negatively correlated with FOXN3 $(P<0.01, \mathrm{R}=-0.322$, fig. 4D). Based on these results, it was hypothesized that FOXN3 functioned as a tumor suppressor in PC by repressing the expression of E2F1 at both mRNA and protein levels. To evaluate the role of E2F1 in the FOXN3mediated inhibition of $\mathrm{PC}$ cell proliferation, the expression of E2F1 was restored in FOXN3overexpressing $\mathrm{PC}$ cells by transfecting an E2F1 expression plasmid in FOXN3-overexpressing PC cell lines. Functional rescue experiment revealed that reintroduction of E2F1 obviously impaired the FOXN3induced suppression of cell proliferation in PC cells (fig. 5A). These results clearly demonstrated that FOXN3 inhibited the proliferation of PC cells by repressing E2F1 expression. As the most common malignant tumor, $\mathrm{PC}$ affects the health of elder men in Europe and becomes an important cause of death ${ }^{[1]}$. As a common clinical heterogeneity multifocal tumor, PC 
A

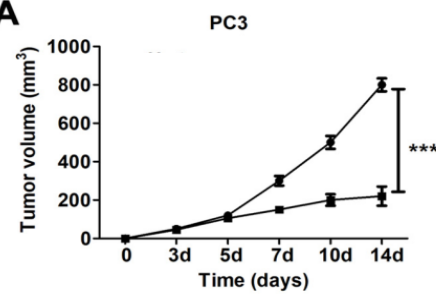

C

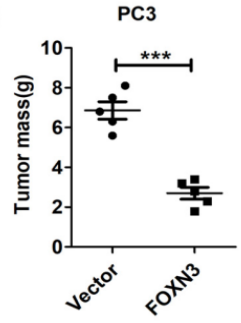

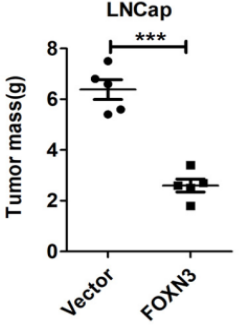

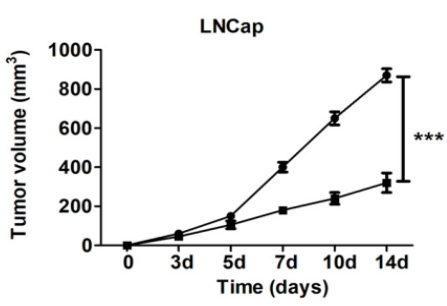

B

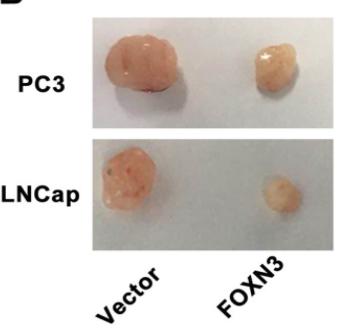

E

PC3

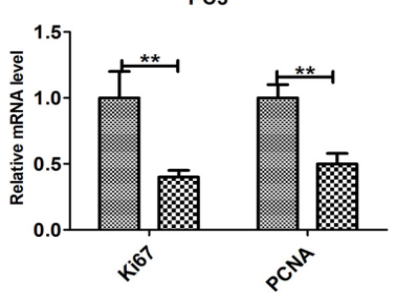

D

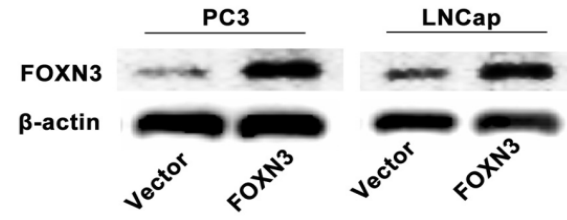

Fig. 3: FOXN3 overexpression inhibited PC cell proliferation in vivo

(A) The volume of xenograft tumors in nude mouse derived from subcutaneous implantation of PC3 or LNCap cells. ( $n=5$ per group). (B) The photograph of the nude mice bearing xenograft tumors at 14 days post-implantation. (C) The tumor weight at 14 days post-implantation. (D) The protein level of FOXN3 in xenograft tumors at 14 days postimplantation. (E) The relative mRNA level of Ki67 and PCNA in xenograft tumors at 14 days post-implantation. $* * P<0.05, * * * P<0.01$.

$$
\text { A }
$$
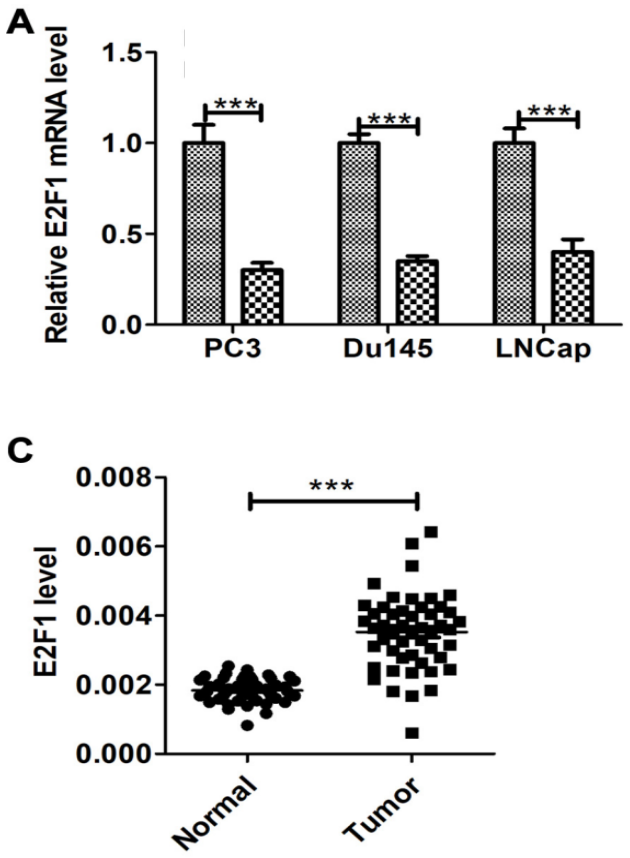

B
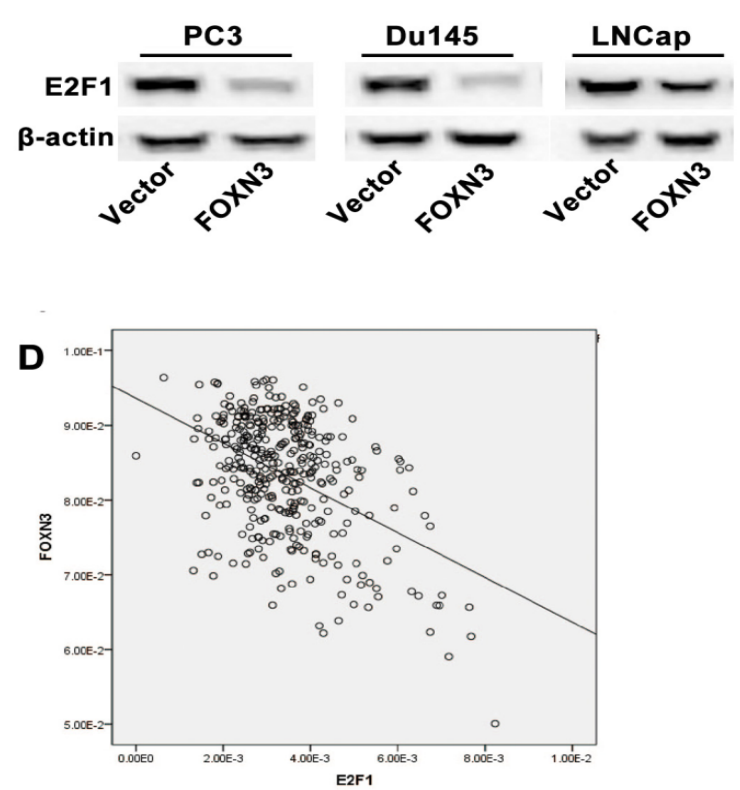

Fig. 4: E2F1 is repressed by FOXN3 as a downstream gene

(A, B) The mRNA and protein level of E2F1 in PC3, Du145 and LNCap cells after infected with lentivirus containing FOXN3 ORF. (C) The mRNA level of E2F1 in human PC tissues compared to non-cancer normal. $N=50$. (D) Correlation analysis of the expression levels of FOXN3 and E2F1 in human PC specimens $(\mathrm{n}=\mathbf{2 5 3}) . * * * P<0.01$. 

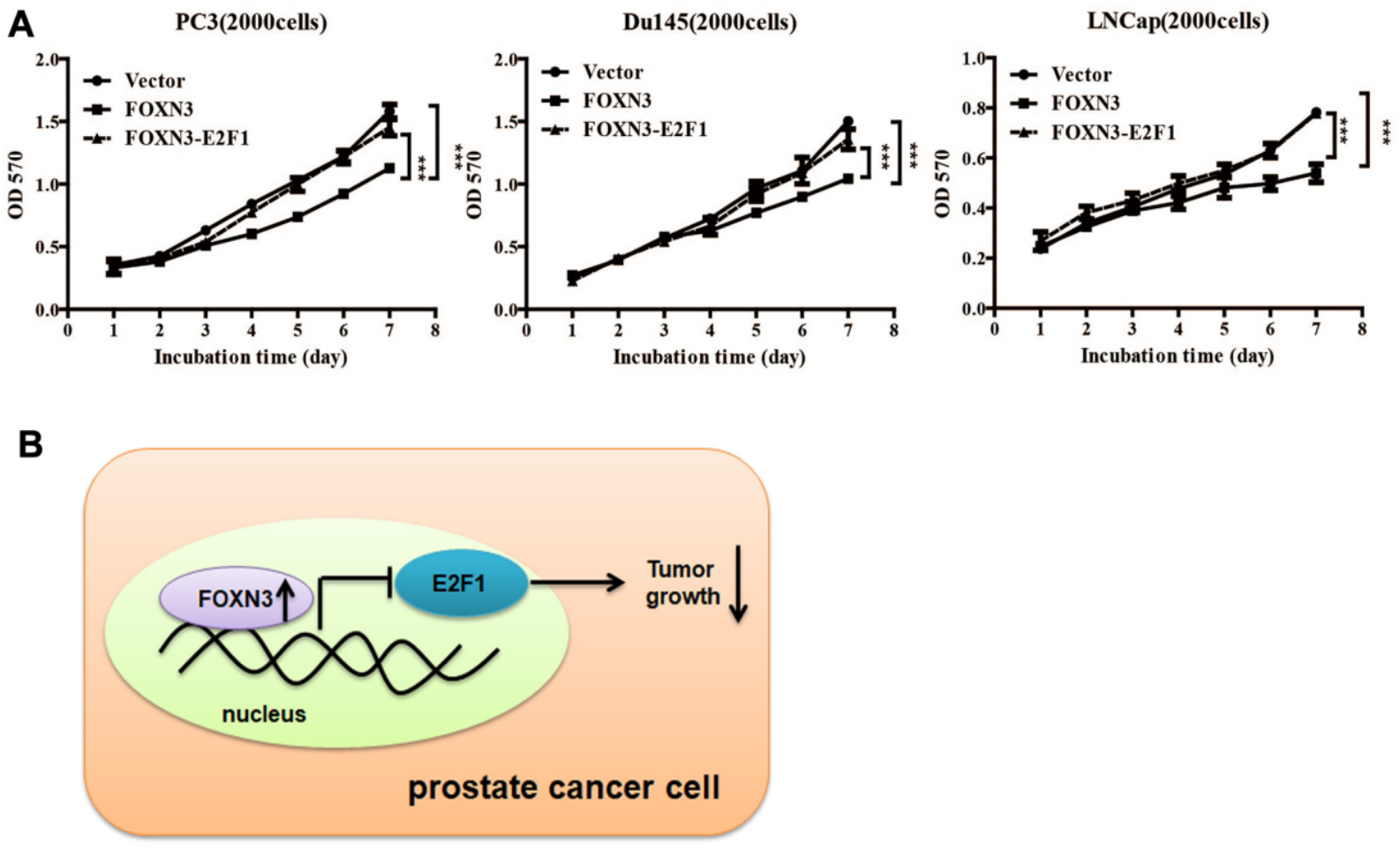

Fig: 5: FOXN3 inhibited PC cell proliferation by repressing E2F1

(A) Effect of FOXN3 overexpression on the cell viability of PC3, Du145 and LNCap cells was abolished by E2F1 overexpression. (B) A proposed model for FOXN3-inhibited E2F1 signaling pathway that leads to an inhibition in PC cell proliferation. $* * * P<0.01$.

has many different behavior types although the incidence of PC is mainly confined to the prostate ${ }^{[19]}$. Some PC subtypes present inert behavior, but has a poor clinical prognosis. More and more clinical pathologic data is used to judge the prognosis of patients with prostate cancer, such as Gleason score, preoperative PSA levels and pathological staging ${ }^{[20]}$. But many studies have shown that patients with similar levels of PSA, Gleason score and pathological staging have different clinical outcomes ${ }^{[21]}$. This shows that the occurrence of prostate cancer is a multi-stage process and has different pathogenesis influencing tumor progression and prognosis. Therefore, in order to understand the progression of $\mathrm{PC}$ and improve the efficiency of treatment and prognosis of PC, finding new diagnostic and therapeutic targets is necessary. More than 100 members of the forkhead box family are classified into 15 subclasses (FOXA through FOXS). As a subclass of FOX family, FOXN has six members: FOXN1, FOXN2 (HTLF), FOXN3 (CHES1), FOXN4, FOXN5 (FOXR1) and FOXN6 (FOXR2) ${ }^{[6]}$. FOXN3 is reported as an indispensable role in cell developmental processes $^{[7]}$. In multiple endocrine neoplasia, FOXN3 protected cells from DNA damge caused by lack of MEN1 under $\mathrm{UV}^{[22]}$. In lung cancer, FOXN3 was repressed by miR-574-5p to promote the procession of cell cycle ${ }^{[23]}$. In hepatocellular carcinoma, cell proliferation was inhibited by FOXN3 via downregulating $\mathrm{E}^{2} \mathrm{~F}^{\left[{ }^{[6]}\right.}$. In colorectal cancer, FOXN3 was demonstrated as a direct transcriptional suppressor of beta-catenin/TCF, which further inhibiting the tumor cells growth and migration ${ }^{[10]}$. These researches showed that FOXN3 functioned as a necessary role in cancer. However, the biological role of FOXN3 in PC remains unclear. Here, FOXN3 was demonstrated as a tumor suppressor in PC for the first time. In our study, The cell proliferation was inhibited by FOXN3in vitro and in vivo via repressing E2F1 (fig. 5B). As a member of E2F family, E2F1 has eight genes (E2F1-E2F8) playing an important role in the $\mathrm{G} 1$ to $\mathrm{S}$ procession of cell cycle $^{[11,14]}$. The expression of E2F1 is closely associated with the high stage of tumor metastasis and the poor prognosis of patients with tumor ${ }^{[24]}$. It has been reported that E2F1 had two opposite functions in cancer. As a tumor suppressor, E2F1 was reported to regulate p53 and its homologue TAp73 activities, and to promote cell apoptosis by a plethora of death pathways activation $^{[25]}$. By contrast, another research showed that E2F1 deregulation enhanced malignant melanoma cells invasion and metastasis independent from its proliferative activity ${ }^{[26]}$. E2F1 regulated CD147 and further promoted PC cell invasion and migration ${ }^{[18]}$. 
The present research showed that E2F1 reversed the FOXN3-induced repression of cell proliferation. Taken together, it was observed that FOXN3 inhibited the proliferation of $\mathrm{PC}$ cells in vitro and in vivo by repressing E2F1 expression. Importantly, FOXN3 may function as a biomarker or a potential target for the prevention and treatment of PC.

\section{Acknowledgments:}

This work was supported by the Natural Science Foundation of Jiangsu Province, China (Grant No. BK2019022920).

\section{Author contributions:}

NingZhang designed experiments, analyzed experimental results and analyzed sequencing data. Qingqi Zeng assisted with Illumina sequencing. Haijun Yang wrote the manuscript.

\section{Conflicts of interest}

All authors declare no conflict of interest.

\section{REFERENCES}

1. Siegel RL, Miller KD, Jemal A. Cancer statistics, 2015. CaCancer J Clin 2015; 65(1):5-29.

2. Jemal A, Lortettieulent J, Ward E, Ferlay J, Brawley OW, Bray F. International Variation in Prostate Cancer Incidence and Mortality Rates. Eur Urol 2012;61(6):1079-92.

3. Huang S, He P, Peng X, Li J, Xu D, Tang Y. Pristimerin Inhibits Prostate Cancer Bone Metastasis by Targeting PC-3 Stem Cell Characteristics and VEGF-Induced Vasculogenesis of BMEPCs. Cell Physiol Biochem 2015; 37(1):253-68.

4. Cai C, Chen Q, Han Z, Zhang Y, He H, Chen J, et al. miR-195 Inhibits Tumor Progression by Targeting RPS6KB1 in Human Prostate Cancer. Clin Cancer Res 2015; 21(21):4922-34.

5. Pati D, Keller C, Groudine M, Plon SE. Reconstitution of a MEC1-independent checkpoint in yeast by expression of a novel human fork head cDNA. Mol Cell Biol 1997;17(6):303746.

6. Sun J, Li H, Huo Q, Cui M, Ge C, Zhao F. The transcription factor FOXN3 inhibits cell proliferation by downregulating E2F5 expression in hepatocellular carcinoma cells. Oncotarget 2016;7(28):43534-45.

7. Li W, Zhang Z, Liu X, Cheng X, Zhang Y, Han X, et al. The FOXN3-NEAT1-SIN3A repressor complex promotes progression of hormonally responsive breast cancer. J Clin Invest 2017;127(9):342140.

8. Schuff M, Rossner A, Wacker SA, Donow C, Gessert S, Knochel W. FoxN3 is required for craniofacial and eye development of Xenopus laevis. Dev Dynam 2007;236(1):226-39.

9. Chang JT, Wang H, Chang K, Chen W, Wen M, Hsu Y. Identification of differentially expressed genes in oral squamous cell carcinoma (OSCC) : Overexpression of NPM, CDK1 and NDRG1 and underexpression of CHES1. Int $\mathrm{J}$ Cancer 2005;114(6):942-9.

10. Dai Y, Wang M, Wu H, Xiao M, Liu H, Zhang D. Loss of FOXN3 in colon cancer activates beta-catenin/TCF signaling and promotes the growth and migration of cancer cells. Oncotarget 2017;8(6):9783-93.

11. Sahin F, Sladek TL: E2F-1 has dual roles depending on the cell cycle. Int J Biol Sci 2010;6(2):116-28.

12. Muller H, Bracken AP, Vernell R, Moroni MC, Christians FC, Grassilli E. E2Fs regulate the expression of genes involved in differentiation, development, proliferation, and apoptosis. Gene Dev 2001;15(3):267-85.

13. Lee TA, Farnham PJ. Exogenous E2F expression is growth inhibitory before, during, and after cellular transformation. Oncogene 2000;19(18):2257-68.

14. Cobrinik D: Pocket proteins and cell cycle control. Oncogene 2005;24(17):2796-2809.

15. Wu L, Timmers C, Maiti B, Saavedra HI, Sang L, Chong GT. The E2F1-3 transcription factors are essential for cellular proliferation. Nature 2001;414(6862):457-62.

16. Hallstrom TC, Nevins JR: Balancing the decision of cell proliferation and cell fate. Cell Cycle 2009;8(4):532-5.

17. Ren Z, Kang W, Wang L, Sun B, Ma J, Zheng C, et al. E2F1 renders prostate cancer cell resistant to ICAM-1 mediated antitumor immunity by NF- $\kappa \mathrm{B}$ modulation. Mol Cancer 2014;13(1):84.

18. Liang Y, Lu J, Mo R, He H, Xie J, Jiang F. E2F1 promotes tumor cell invasion and migration through regulating CD147 in prostate cancer. Int J Oncol 2016; 48(4):1650-8.

19. Han M, Brannigan RE, Antenor JAV, Roehl KA, Catalona WJ. Association of hemospermia with prostate cancer. J Urol 2004;172(6):2189-92.

20. Borley N, Feneley MR. Prostate cancer: diagnosis and staging. Asian Journal of Andrology 2009, 11(1):74-80.

21. Sfoungaristos S, Perimenis P. PSA density is superior than PSA and Gleason score for adverse pathologic features prediction in patients with clinically localized prostate cancer. Cuaj-Can Urol Assoc 2012;6(1):46-50.

22. Busygina V, Kottemann MC, Scott KL, Plon SE, Bale AE. Multiple Endocrine Neoplasia Type 1 Interacts with Forkhead Transcription Factor CHES1 in DNA Damage Response. Cancer Res 2006;66(17):8397-403.

23. Li Q, Li X, Guo Z, Xu F, Xia J, Liu Z. MicroRNA-574-5p Was Pivotal for TLR9 Signaling Enhanced Tumor Progression via Down-Regulating Checkpoint Suppressor 1 in Human Lung Cancer 2012;7(11):e48278.

24. Kang K, Lee M, Song J, Jeong J, Kim Y, Lee C. Overexpression of goosecoid homeobox is associated with chemoresistance and poor prognosis in ovarian carcinoma. Oncol Rep 2014;32(1):189-98.

25. Engelmann D, Putzer BM. Translating DNA damage into cancer cell death-A roadmap for E2F1 apoptotic signalling and opportunities for new drug combinations to overcome chemoresistance. Drug Resist Update 2010;13(4):119-31.

26. Alla V, Engelmann D, Niemetz A, Pahnke J, Schmidt A, Kunz M. E2F1 in Melanoma Progression and Metastasis. J Nat Cancer Inst 2010;102(2):127-33.

This is an open access article distributed under the terms of the Creative Commons Attribution-NonCommercial-ShareAlike 3.0 License, which allows others to remix, tweak, and build upon the work non-commercially, as long as the author is credited and the new creations are licensed under the identical terms

This article was originally published in a special
issue, "Biomedical Research in Healthcare Setting"
Indian J Pharm Sci 2020:82(3)Spl issue5;137-143

